\title{
Genome-wide identification and characterization of bZIP transcription factors and their expression profile under abiotic stresses in Chinese pear (Pyrus bretschneideri)
}

\author{
Muhammad Aamir Manzoor', Muhammad Mudassar Manzoor², Guohui Li', Muhammad Abdullah', Wang Han', \\ Han Wenlong ${ }^{1}$, Awais Shakoor ${ }^{3}$, Muhammad Waheed Riaz ${ }^{4}$, Shamsur Rehman ${ }^{5}$ and Yongping Cai ${ }^{i^{*}}$
}

\begin{abstract}
Background: In plants, basic leucine zipper transcription factors (TFs) play important roles in multiple biological processes such as anthesis, fruit growth \& development and stress responses. However, systematic investigation and characterization of bZIP-TFs remain unclear in Chinese white pear. Chinese white pear is a fruit crop that has important nutritional and medicinal values.

Results: In this study, 62 bZIP genes were comprehensively identified from Chinese Pear, and 54 genes were distributed among 17 chromosomes. Frequent whole-genome duplication (WGD) and dispersed duplication (DSD) were the major driving forces underlying the bZIP gene family in Chinese white pear. bZIP-TFs are classified into 13 subfamilies according to the phylogenetic tree. Subsequently, purifying selection plays an important role in the evolution process of PbbZIPs. Synteny analysis of bZIP genes revealed that 196 orthologous gene pairs were identified between Pyrus bretschneideri, Fragaria vesca, Prunus mume, and Prunus persica. Moreover, cis-elements that respond to various stresses and hormones were found on the promoter regions of PbbZIP, which were induced by stimuli. Gene structure (intron/exon) and different compositions of motifs revealed that functional divergence among subfamilies. Expression pattern of PbbZIP genes differential expressed under hormonal treatment abscisic acid, salicylic acid, and methyl jasmonate in pear fruits by real-time qRT-PCR.

Conclusions: Collectively, a systematic analysis of gene structure, motif composition, subcellular localization, synteny analysis, and calculation of synonymous (Ks) and non-synonymous (Ka) was performed in Chinese white pear. Sixty-two bZIP-TFs in Chinese pear were identified, and their expression profiles were comprehensively analyzed under ABA, SA, and MeJa hormones, which respond to multiple abiotic stresses and fruit growth and development. PbbZIP gene occurred through Whole-genome duplication and dispersed duplication events. These results provide a basic framework for further elucidating the biological function characterizations under multiple developmental stages and abiotic stress responses.
\end{abstract}

Keywords: PbbZIP gene family, Phylogenetic analysis, Expansion, Expression analysis, Stress response

\footnotetext{
* Correspondence: ypcaiah@163.com

'School of Life Sciences, Anhui Agricultural University, Hefei 230036, China

Full list of author information is available at the end of the article
}

(c) The Author(s). 2021 Open Access This article is licensed under a Creative Commons Attribution 4.0 International License, which permits use, sharing, adaptation, distribution and reproduction in any medium or format, as long as you give appropriate credit to the original author(s) and the source, provide a link to the Creative Commons licence, and indicate if changes were made. The images or other third party material in this article are included in the article's Creative Commons licence, unless indicated otherwise in a credit line to the material. If material is not included in the article's Creative Commons licence and your intended use is not permitted by statutory regulation or exceeds the permitted use, you will need to obtain permission directly from the copyright holder. To view a copy of this licence, visit http://creativecommons.org/licenses/by/4.0/ The Creative Commons Public Domain Dedication waiver (http://creativecommons.org/publicdomain/zero/1.0/) applies to the data made available in this article, unless otherwise stated in a credit line to the data. 


\section{Background}

Transcription factors (TFs) play crucial roles in the regulation and biological processes under various environmental stress [1, 2]. The basic leucine zipper (bZIP) transcription factors family member is one of the most diverse and largest transcription factors in eukaryotes [3]. The length of the domain is $60-80$ amino acids containing two regions with various functions; the basic region is highly conserved and consists of 16 amino acid residues with specific DNA binding $(\mathrm{N}-\times 7-\mathrm{R} / \mathrm{K}-\times 9)$ [4]. On the other hand, the leucine zipper is less conserved and composed of several helical structures, which are responsible for dimerization stability and specificity (homo/heterodimerization) [5].

Abiotic stress resistance in plants is mediated by the hormone $\mathrm{ABA}$, which regulates many stress-responsive genes and confers abiotic stress tolerance in plants [6]. The group A AtbZIP protein includes ABA-responsive element-binding proteins (AREB) or ABRE binding factors $(\mathrm{ABF})$ that have been functionally identified as major regulators of ABA-dependent gene expression and the abiotic stress response $[1,6]$. Phosphorylation of category III SnRK2s activates AREB1/ABF2, AREB2/ABF4, $A B F 1$, and $A B F 3$ in Arabidopsis, controlling the expression of their downstream genes [7, 8]. AtbZIP17, AtbZIP60, and AtbZIP28 proteins play significant roles in ER stress responses [9]. In Arabidopsis AtbZIP28, AtbZIP17 genes are activated by salt stress and function as a salt stress sensor/transducer [10]. Salt treatment increased the transcription of AtbZIP1, MtbZIP2, and MtbZIP26 from A. thaliana and Medicago truncatula, resulting in an increase in salt stress resistance [11-13]. The bZIP family members have been comprehensively identified and functionally characterized in various plants such as A. thaliana (75 genes) [1], B. napus (247 genes) [14], Solanum lycopersicum (69 genes) [15], $B$. distachyon (96 genes) [16], Oryza sativa (89 genes) [3], Zea mays (125 genes) [17], Glycine max (131 genes) [18], Coniothyrium minitans, (34 genes) [19], Vitis vinifera (55 genes) [20], Malus domestica (116 genes) [21], Fragaria vesca (50 genes) [22], Sorghum bicolor (92 genes) [23] and Prunus persica (47 genes) [24], Ricinus communis L. [25].

Plant bZIP proteins plays important roles in plant growth and diverse biological functions, including somatic embryogenesis [26], seed germination [20, 27], cell elongation [28], vascular development [29], floral induction and growth [30], unfolded protein response [31], and photomorphogenesis [32]. Plant bZIP transcription factors have also been involved in a variety of abiotic/biotic stress-related including hormone signaling and sugar response [33-36], light response [37, 38], pathogen infection [39, 40], cold signaling [41, 42], osmotic stresses [43], heat stress [44] and so on. Generally, ABA- responsive binding factor (ABRE) and binding protein (AREB) are associated with ABA and stress-related factors [45]. In rice, OsbZIP71 acts as ABA-mediated salt and drought tolerance [46]. ZmbZIP17 in maize is also highly induced with the interaction of ABA (cis-element ABRE) [45]. SlbZIP33 (SlAREB1) was implicated in stress-induced responses in tomato and acted as critical components in regulating the expression of important metabolic pathway-related genes and metabolic programming during fruit ripening $[47,48]$.

Chinese pear (Pyrus. bretschneideri) fruit crop is cultivated worldwide, especially in China, and has become most popular or essential due to its high medicinal and nutritional value $[49,50]$. Although bZIP members have been identified in various model plants, this gene family has not been comprehensively studied in Pyrus. bretschneideri. However, a comprehensive and systematic analysis of the bZIP family has been identified in Chinese pear. Finally, in this study, 62 PbbZIP genes were identified and classified into 13 groups according to the phylogenetic tree. The evolutionary relationship/history, chromosomal distribution, conserved domain, gene duplication, gene structure analysis, conserved motif, microsynteny relationship, cis-elements on the promoter regions, $\mathrm{ka} / \mathrm{ks}$ value analysis, and other important information were investigated in PbbZIP genes. The expression profiles of PbbZIP genes in different fruit development stages were analyzed using RNA-seq data. Afterward, the expression patterns of 12 genes were examined in response to Abscisic acid (ABA), salicylic acid (SA), and methyl salicylate (MeJA) by conducting qRTPCR. These results would provide specific knowledge and evolution in Chinese pear for future studies.

\section{Results}

Identification of bZIP gene family in Chinese white pear The pear bZIP transcription factors family members were identified by using Hidden Markov Model (HMM) and Blast method. A systematic approach bZIP domain (PF00170) analysis was also performed using the Pfam database. Initially, pear bZIP family members were identified, and their sequences were downloaded from the pear genome. Finally, identified candidate genes were briefly analyzed and verified the bZIP domain. Subsequently, 62 members of this family encoding complete bZIP DNA binding domain were selected for sequence alignment and for Further analysis. Nomenclature of these identified genes was given based on chromosome position and renamed all genes PbbZIP1 to PbbZIP62 (Table S2). The length of amino acid varied from 141 AA (PbbZIP41) to 1726 AA (PbbZIP16), with a crossponding isoelectric point (IP) varied from 4.96 (PbbZIP28) to 10.51 (PbbZIP50). The molecular weight of these genes ranged from $16,313.4 \mathrm{kDa}$ to $194,044.9$ 
kDa. Detailed information, including gene i.d, given name, molecular weight, isoelectric point, gene size, and chromosomal distribution, are mentioned in Table S2.

\section{Phylogenetic analysis}

To further understand the evolutionary relationship, difference, and similarities of bZIP transcription factor between pear (62 genes), Arabidopsis (75 genes), grapes (55 genes), and poplar (86 genes). The unrooted phylogenetic tree was generated through neighbor-joining methods (NJ-M), and the maximum likelihood method (ML-M). For future study neighbor-joining tree was used to perform a phylogenetic characterization in Chinese pear with Arabidopsis (Fig. 1 and S2). Finally, the phylogenetic tree was visualized through itol software and categorized into 13 subfamilies (A, B(F/Ara), C(D/Ara), D, $\mathrm{E}(\mathrm{B} /$ Ara $), \mathrm{F}(\mathrm{H} /$ Ara $), \mathrm{G}, \mathrm{H}(\mathrm{E} /$ Ara $), \mathrm{I}(\mathrm{I} /$ Ara $), \mathrm{J}(\mathrm{G} /$ Ara $)$, $\mathrm{K}(\mathrm{A} /$ Ara $), \mathrm{L}(\mathrm{C} / /$ Ara $), \mathrm{M}(\mathrm{S} /$ Ara $)$. These groups were classified according to the Arabidopsis groups, in subfamily $D$ and $G$ absent in pear, but in the subfamily, A only contained pear and poplar genes except Arabidopsis may be due to functional divergence or these subfamilies indicating that specific functions. Furthermore, an inspection of the phylogenetic tree, maximal PbbZIPs members contained in subfamily A (13) and minimal in subfamily E-F (2) (Fig. 1). Subfamily C, D, G, and H formed clades without pear genes indicating that these clades might be specific for poplar and Arabidopsis. Gene loss and gain mechanisms occurred in evolutionary mechanisms and caused functional divergence.

\section{Gene ontology (GO) analysis and subcellular localization of PbbZIP}

GO annotation analysis was performed for the prediction of various functions in PbbZIP members. Gene ontology such as cellular components, biological processes, and molecular functions. Additionally, 62 PbbZIP proteins were grouped into 44 functional groups according to protein similarities under the three basic groups. In the ontology of biological process, we evaluate that the highest percentage were involved in the biosynthetic process (16.39\%) and cellular nitrogen compound metabolic process (16.39\%). Among the PbbZIP, 13.98, 12.87, $10.33 \%$ of genes showed potential involvement in signal

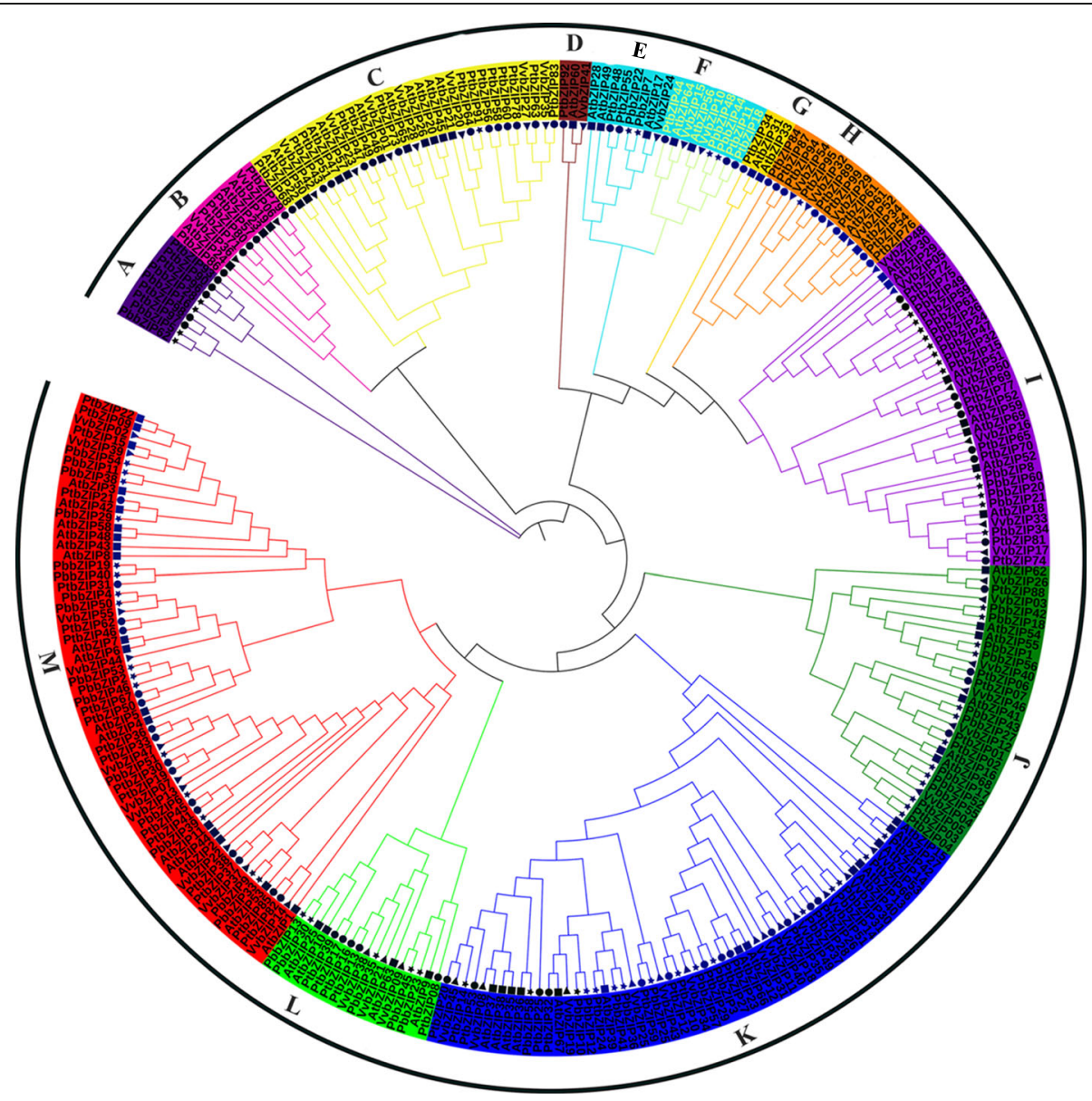

Fig. 1 The phylogenetic tree of bZIP genes in Chinese pear, Arabidopsis, grapes, and poplar. Different color indicates different subfamilies (A-M) 
transduction, response to stress, and hemostatic process, respectively. Also, some genes are involved in development $(0.43 \%)$, the immune system $1.88 \%$, cell deafferentation $0.86 \%$, and anatomical structure $5.50 \%$ (Fig. 2). In the ontology of molecular functions, PbbZIPs genes the highest percentage are involved in nucleic acid binding transcription function activity, and DNA binding $40.46 \%$, followed by protein binding transcription factor $5.59 \%$, enzyme regulator activity $0.40 \%$, RNA binding $0.40 \%$, ion binding $3.36 \%$, hydrolase activity $1.61 \%$, protein binding $1.80 \%$, signal transducer activity $0.40 \%$, kinase activity $0.40 \%$.

Moreover, cellular component ontology suggested that also contain cellular components followed as intracellular, cell, and organelle found are same and high percentage $17.74 \%$ as compared to another cytoplasm (14.65\%), cytosol $(2.58 \%)$, nuclear chromosome $(0.16 \%)$, nucleoplasm $(1.36 \%)$, chromosome $(0.16 \%)$, protein complex (0.86), and endoplasmic reticulum (0.92\%) (Table S3). Subcellular localization was predicted by using CELLOGO tool software. These results indicate that most of the PbbZIP genes (96.8\%) were localized in nuclear, while remained genes were involved in cytoplasm and chloroplast (Table S3).

\section{Gene structure analysis and identification of conserved motif}

Structural analysis was performed by comparing general feature format (GFF3) files of each bZIP gene in Chinese white pear to understand the phenomena of structural diversity. The composition and position of introns/exons identified using Gene structure display server and compare the gene structure among different or same subfamily. In PbbZIP genes contain maximum introns/ exons (18/19) (Fig. 3A). These results revealed that 5 PbbZIP genes (PbbZIP11, 40, 19, 4, and 46) had no introns in their gene structures, while remained PbbZIP genes contained 1 to 18 introns.

Additionally, the PbbZIPs gene in subfamily A contained the largest number of exons/introns; for example, PbbZIP16 had 19-exons/18-introns. On the other hand, the number of introns/exons variant also in the same subfamily, such as PbbZIP31, had 4-exons/4-introns. These results revealed that the divergence of introns/ exons structure in the PbbZIPs family might be related to different functions.

Conserved motif analysis was performed through the protein sequence of PbbZIP genes using a MEME server. Distribution and composition of conserved PbbZIP motif ratio similar among the subfamily, supporting the results of the phylogenetic tree. A total of twenty (20) motifs were predicted in PbbZIP genes, and motif contained in PbbZIP members varied within the subfamily. PbbZIP genes were divided into different subfamilies $(\mathrm{M}, \mathrm{L}, \mathrm{J}, \mathrm{K}$, F, I, A, and E) according to the phylogenetic tree and divergence of motifs. The subfamily-I encoded a large number of conserved motifs. The number of motifs was

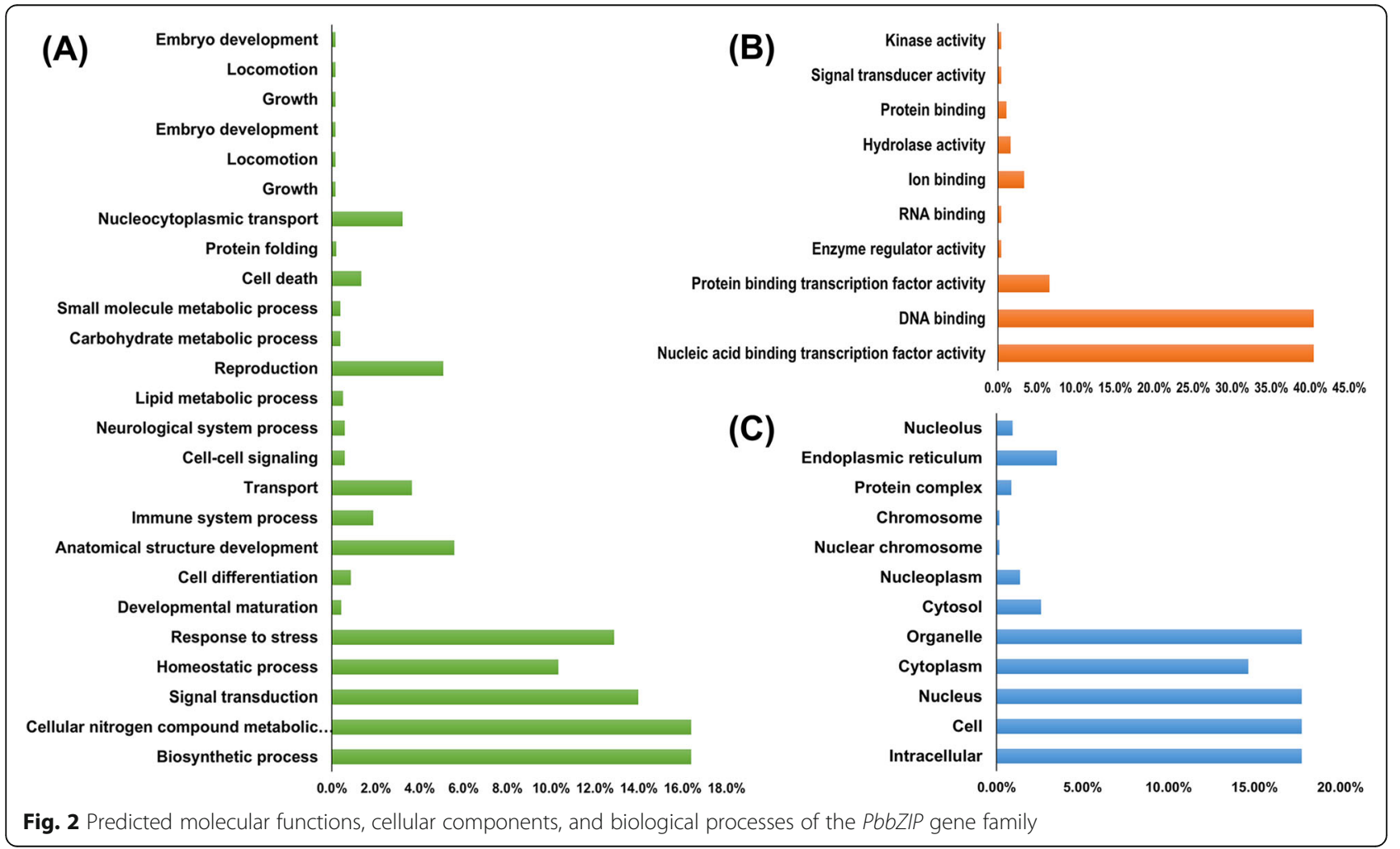




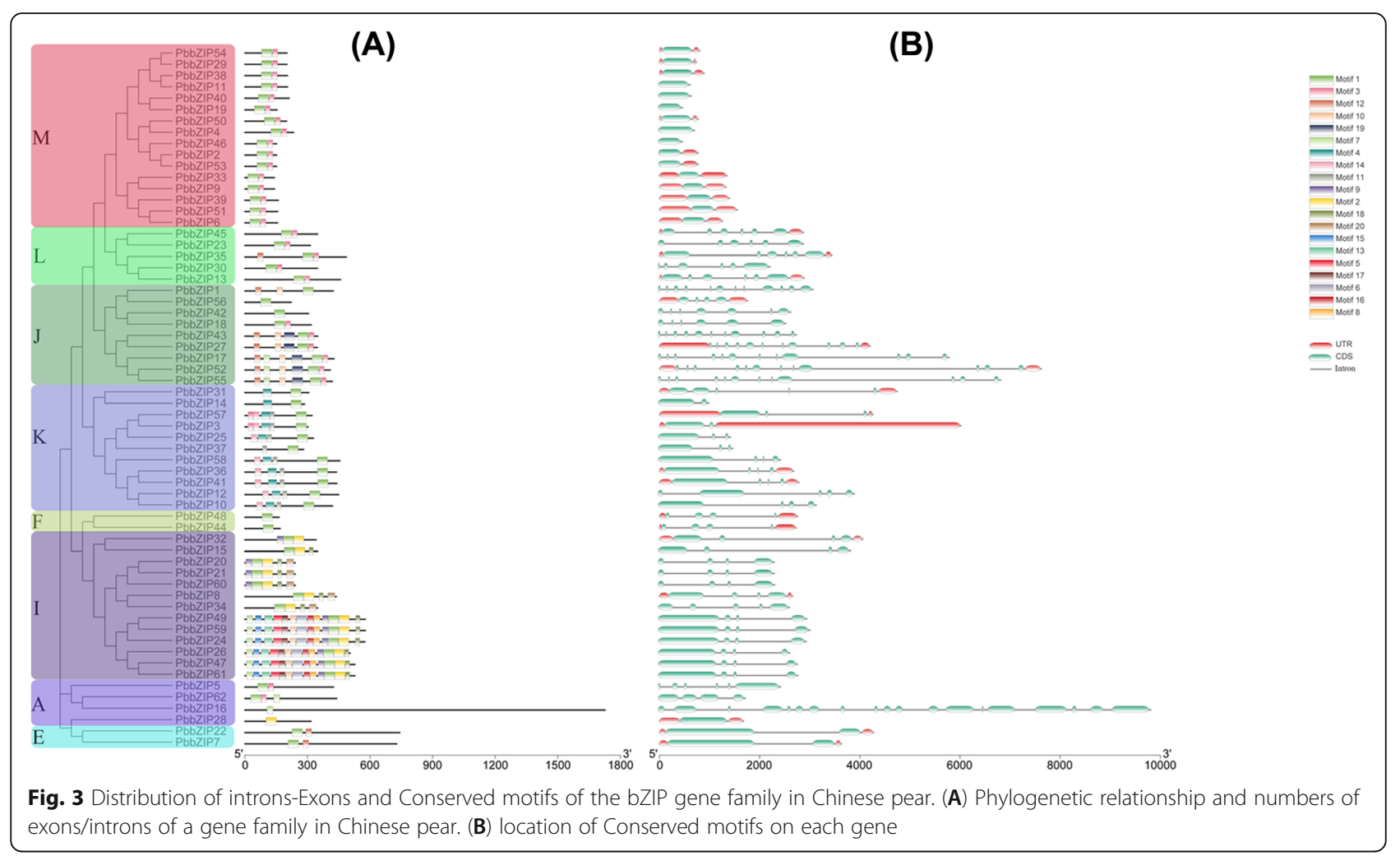

variant among different and same subfamilies. The number of motifs was different between these subfamilies $M$ (maximum number of motifs 2), L (maximum number of motifs 3), J (number of motif 9), K (maximum number of motif 6), F (maximum number of motif 1), I (maximum number of motif 13), A (maximum number of motif 3 ) and E (maximum number of motif 2) (Fig. 3B). Conserved motif two highly contribution is mostly genes. The presence of numerous motifs in PbbZIP members indicates that similar duplication events. In Chinese pear (Pyrus bretschneideri), among 65 bZIP members, 50 residues conserved domain (motif 2). The presence of a conserved motif between the same bZIP protein, especially within the subfamily, likely reflects the similarities in functions. These proteins conserved different motifs in the same subfamily reflect that they have different functions (Table S4).

\section{Identification of gene duplication events, expansion, and chromosomal distribution}

To elucidate the origin of the bZIP TFs gene family in Chinese pear, four types of duplication (WGD-whole genome duplication, TD-tandem duplication, TRDtransposed duplication, and DSD- dispersed duplication) were carryout (Fig. 4B). A total of 87 duplicated pairs were reported in Chinese pear followed by DSDs (41 gene pairs), WGDs (42 gene pairs), TDs (2 gene pairs), and TRDs ( 2 gene pairs), indicating that the expansion of the gene family (Fig. 4A). Expansion of gene family basically occurred by the whole genome duplication and dispersed duplication [51]. In the study, identified tandem and dispersed duplication to elucidate expansion of bZIP genes in Pyrus bretschneideri. Simultaneously, these results also indicate the complicated duplication mechanism of the PbbZIP family. All duplication modes (DSDs, WGDs, TDs, and TRDs) were involved in the evolution and expansion of PbbZIP genes. In pear, $41 \%$ of genes showed dispersed duplication (DSD), while $2 \%$ tandem duplication (TD) suggesting that dispersed duplication events highly contribute to the expansion and evolution of the PbbZIP genes family as compared to tandem duplication events (Table S5). The number of duplicated genes was different on duplication mode such as WGD (maximum eight duplicated genes on chromosome 15), DSD (maximum six duplicated genes on scaffold), TD (maximum one duplicated genes on chromosome 15 and scaffold respectively), TRD (maximum one duplicated genes on chromosome 8 and scaffold respectively) (Table S5). To understand the localization of PbbZIP genes on a different chromosome number, all genes of pear are mapped except that gene located on scaffold according to the pear genome. As a result of the 62 PbbZIP gene, 54 genes were located on the chromosome, and eight genes were located on the scaffold. Moreover, maximum 9 genes are traced on chromosome 15 . On the other hand, chromosome 16 minimum carried one gene (Fig. S1 and Table S2). 


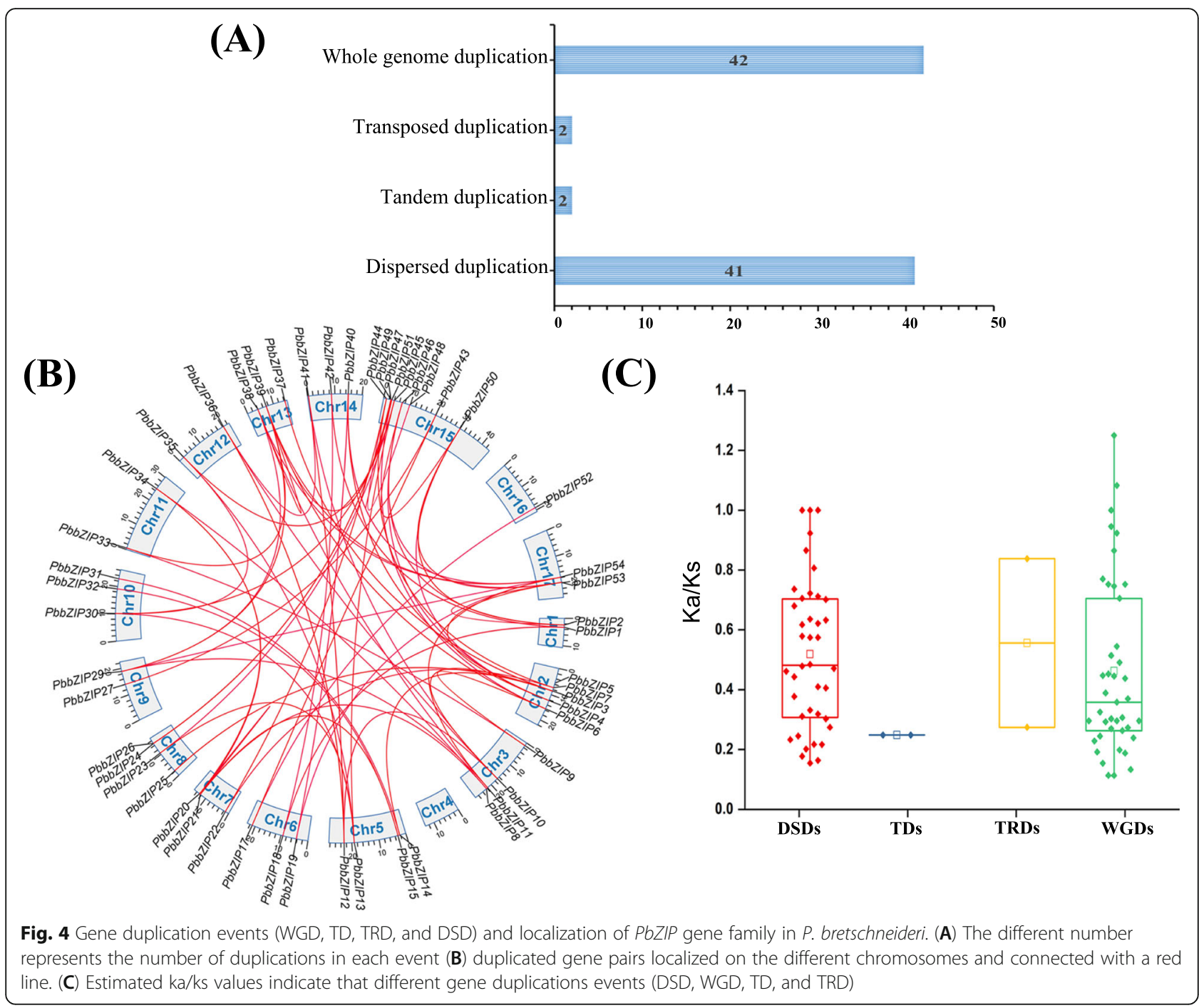

\section{Estimation of $\mathrm{Ka} / \mathrm{Ks}$ analysis}

To determine the evolutionary date in gene duplication events, we calculate synonymous (Ks) and nonsynonymous (Ka) values for 87 duplicated gene pairs. The $\mathrm{Ka} / \mathrm{Ks}$ value ranged from 1.24 to 0.21 , as shown in Table S5. In general, $\mathrm{Ka} / \mathrm{Ks}$ value greater than one was suggested that positive selection, $\mathrm{Ka} / \mathrm{Ks}$ less than 1 suggested purifying selection, and $\mathrm{Ka} / \mathrm{Ks}=1$ suggest neutral selection [52, 53]. In our study, all PbbZIP gene pairs $\mathrm{Ka} / \mathrm{Ks}$ value less than 1 , indicating that these genes are primarily undergone in purifying selection. On the other hand, four genes $\mathrm{ka} / \mathrm{ks}$ value is equal to 1 , indicating that positive selection (Fig. 4C). We also calculated the ka/ks value in DSD, TD, TRD, and WGD. These gene pairs PbbZIP40-PbbZIP19 had (ka/ks 1.0827), and PbbZIP10PbbZIP12 (ka/ks 1.2498$)$ had higher $\mathrm{Ka} / \mathrm{Ks}$ value indicates that this gene family complicated evolutionary history.

\section{Synteny analysis of PbZIP genes}

The collinearity relationship is constructed among homology in other species to illustrate the collinearity association of PbZIP genes between Fragaria vesca, Prunus mume, and Prunus persica. The collinearity relationship between Pyrus bretschneideri, Fragaria vesca, Prunus mume, and Prunus persica showed a total of 196 orthologous pairs. The orthologous pairs of PbbZIP genes with other species, including Pyrus bretschneideri-Fragaria vesca (63 pairs), Pyrus bretschneideri-Prunus mume (61 pairs), and Pyrus bretschneideri-Prunus persica (72 pairs) Table S6. Finally, these results indicate that the close similarities relationship with other orthologous and phylogenetic relationships (Fig. 5).

\section{Cis-elements analysis}

Promoter activities play a vital role in the regulatory mechanism and different types of gene functions [54]. 


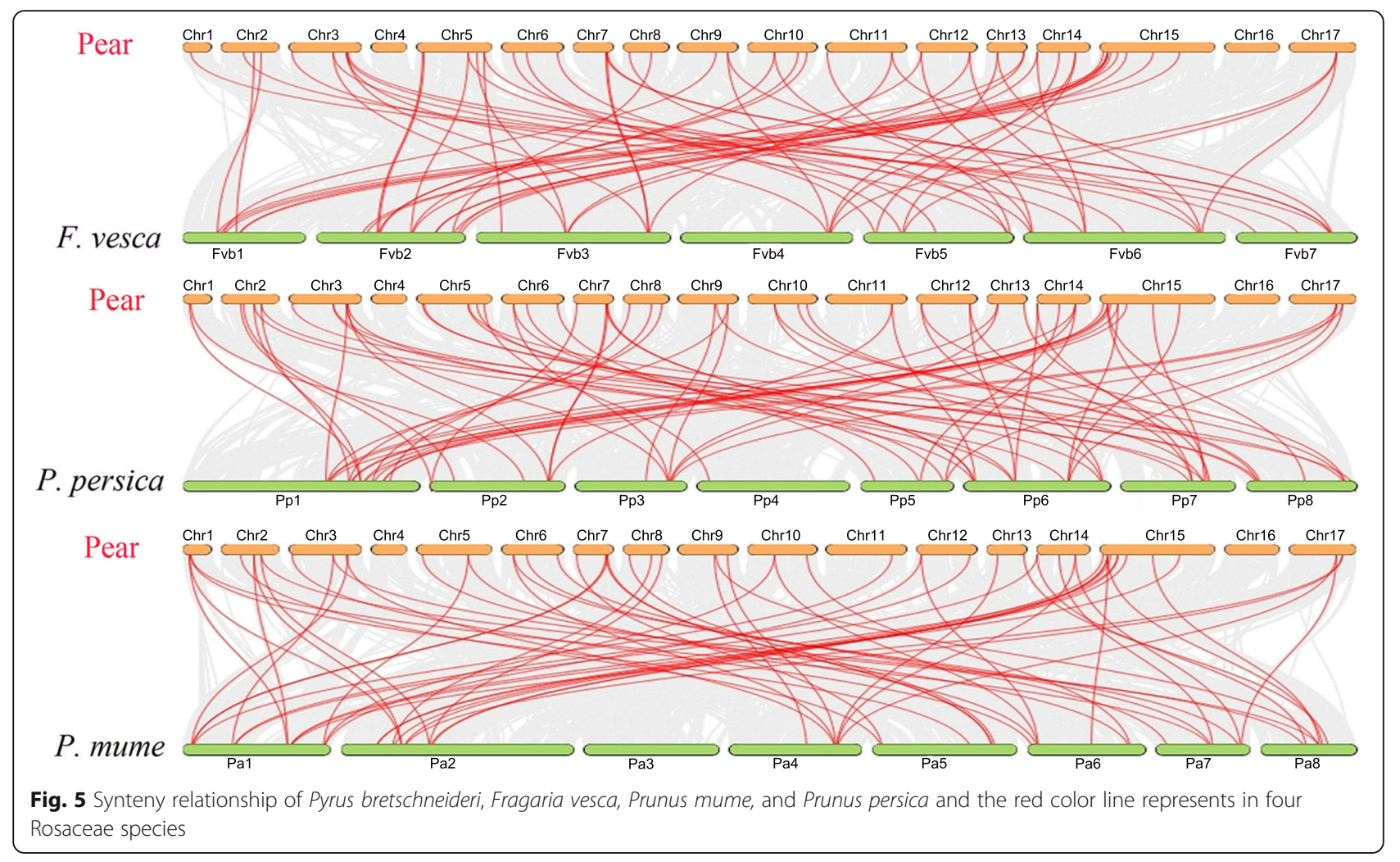

The cis-elements analysis on promoter regions was analyzed to estimate the metabolic network, including stress response, tissue-specific expression, multi stimulusresponse, and other environmental conditions [53, 55]. Trans-acting elements and cis-regulatory elements play a potential mechanism for the increase and decrease of the expression of any gene. Cis-elements were obtained on the promoter regions of PbbZIP genes to understand the potential role and mechanism. We observed the highest amount of cis-elements on the promoter regions through plantCare database. Furthermore, cis-elements were classified into three basic categories: (1) stress response (biotic/abiotic) (2) plant growth/development (3) phytohormones responsive (Fig. 6). In-plant growth/development, mostly PbbZIPs genes contain as regulatory elements such as Box 4, MRE, G-Box (plant growth response in light), CAT-box (meristem related), 02-site (zein metabolism related). In stress related cis-elements including MBS (light responsive), LTR (low temperature related) and ARE (responsible anaerobic induction). Subsequently, in phytohormones related class identified large number of cis-elements including P-box and GARE-motif (gibberellin response elements). Among all these three classes (stress response, plant growth/development, phytohormones responsive) the dominant portion G-box (28\%), CAT-box and 02-site (40\%) and TGACG-motif and CGTCA-motif (30\%) respectively. These results imply that different type of cis-elements in the same types of PbbZIP genes might have various functions (Table S7). Further, TCA-element involved in salicylic acid (SA) response, TGACG-motif and CGTCA had involve in as a methyl jasmonate (MeJA) responsive cis-elements and ABRE had abscisic acid (ABA) responsive cis-elements. These hormones (ABA, SA and MeJA) were involved in senescence and fruit ripening $[56,57]$. These results suggesting that bZIP family may be associated with hormonal changes and involved in pear fruit senescence, ripening and maturation.

\section{Expression patterns of PbZIP genes in different fruit development stages}

Based on RNA-seq data in pear fruit at different development stages, we investigate the expression profile of PbZIP genes (Table S8). The RNA-seq data carried out from previous studies conducted in 7 fruit development stages ((stage1-15DAB, stage2-30DAB, stage3-55DAB, stage4-85DAB, stage5-115DAB, stage6- mature stage, and stage7-fruit senescence stage). The microarray data was used to investigate the spatiotemporal expression profile of PbZIP members in different fruit stages. Fragments per kilobase million (FPKM) values were used to analyze the gene expression pattern. These results showed that PbZIPs expressed patterns increase and decreased in different development stages. PbZIP49, PbZIP59 genes were abundantly expressed in stage-7 development stages, implying that maybe these genes play 


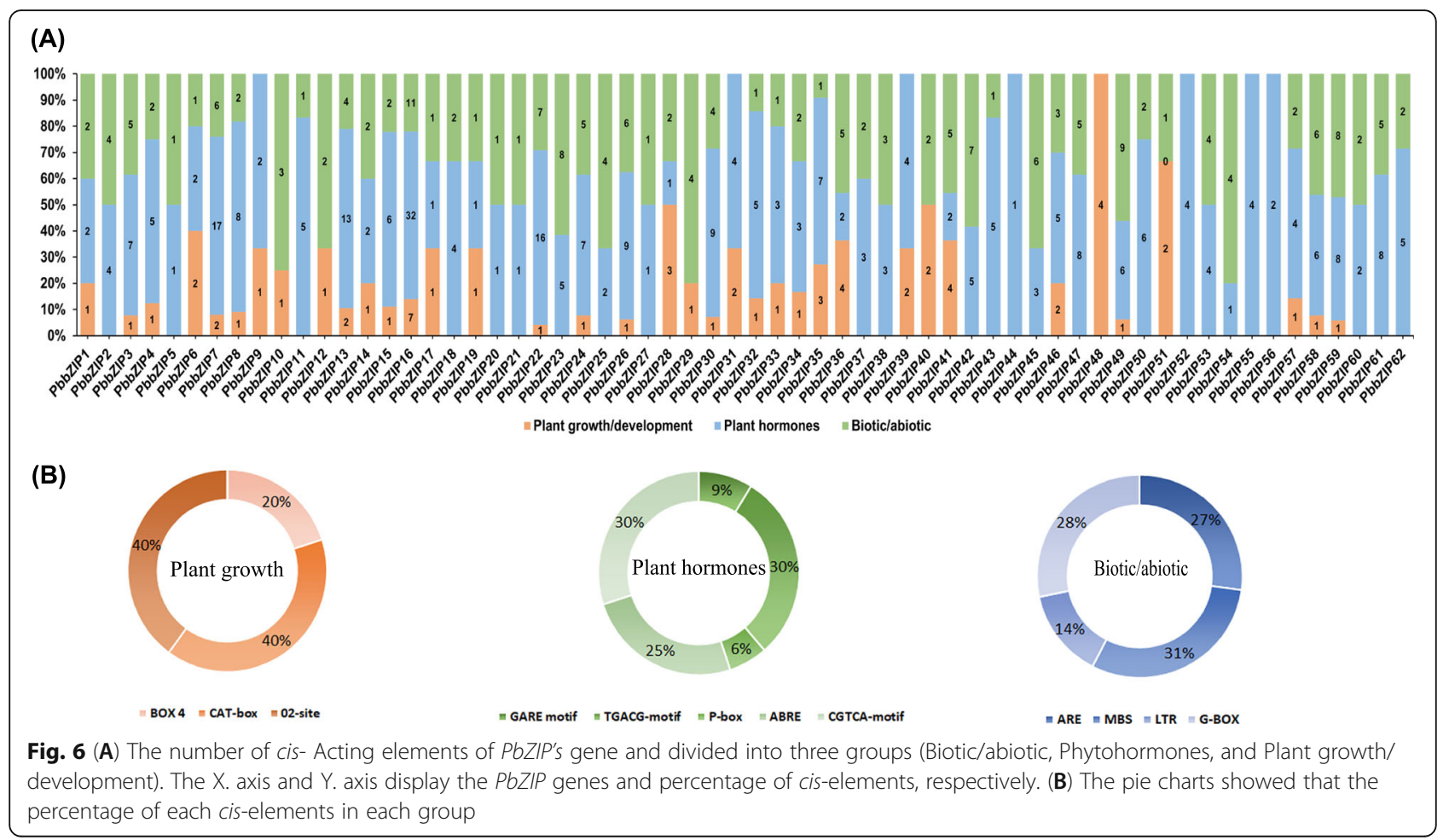

a critical role in fruit ripening and development. On the other hand, the expression profile of 2 genes (PbZIP39, PbZIP51) increased, and three genes (PbZIP33, PbZIP6, and PbZIP9) decrease during fruit development stages, suggesting that they may play an important role in early and later stages. The expression pattern in the development stage 1 and stage 2 is higher than that of other developmental stages of pear fruits (Fig. 7).

\section{Expression analysis of PbZIP genes under hormonal treatment}

To further examine the expression pattern of PbZIP under hormonal treatment stresses (ABA, SA, and MeJA). Twelve genes were randomly selected after the spraying on pear fruit at different time duration $(1 \mathrm{~h}, 2 \mathrm{~h}$, $3 \mathrm{~h}$ ). Finally, the expression patterns were analyzed using qRT-PCR. Application of exogenous hormonal spray two types of expression were observed inhibit and induced. Under the ABA (Abscisic acid) hormones treatment, most of the genes (PbZIP3, 13, 58, 49, 16, and 26) showed the highest expression pattern on $3 \mathrm{~h}$, PbZIP27,18,30,22 and 61 genes peak expression on $2 \mathrm{~h}$, and only one gene (PbZIP31) showed high expression as compared to control (CK) at 39 DAF. All genes showed significant expression under the ABA treatment (Fig. 8A). In SA (salicylic acid) treatment, we found that PbZIP18, $30,13,16$, and 22 were achieved the highest expression pattern at $1 \mathrm{~h}$. While PbZIP27, 3, 49, 26, and 61 strongly upregulated at $3 \mathrm{~h}$. on the other hand, two genes
(PbZIP58 and 31) significantly expressed at $2 \mathrm{~h}$ (Fig. 8B). In case of MeJA (methyl jasmonate) treatment, genes have also seen the same trend as were in Abscisic acid and salicylic acid except for PbZIP22. Five genes, namely PbZIP18, 30, 49,16, and 61 upregulate expression at $3 \mathrm{~h}$, while the PbZIP22 gene inhibits expression at $1 \mathrm{~h}, 2 \mathrm{~h}$ and $3 \mathrm{~h}$. The expression PbZIP27, 13, 31, and 26 significantly achieved expression at $1 \mathrm{~h}$ and 2 genes (PbZIP58, 3 ) at $2 \mathrm{~h}$ higher as compared to control. (Fig. 8C).

\section{Discussion}

The basic leucine zipper (bZIP) transcription gene family has been identified and comprehensively studied in many plants such as Arabidopsis, Oryza sativa, Zea mays, Glycine max, Coniothyrium minitans, Vitis vinifera, Ricinus communis L., Malus domestica, Fragaria vesca, Sorghum bicolor, B. napus, Solanum lycopersicum, B. distachyon, Prunus persica, and evidence indicates that these genes participate in different physiological and developmental processes in plants. In this study, we identified and analyzed 62 genes in Chinese white pear and investigated their expression patterns on different fruit developmental stages under hormonal stress. Synteny analysis, evolutionary history, cis-elements analysis, chromosol location gene structure, and gene duplication were examined. Although the bZIP family has been identified and characterized in many crops, yet systematic and comparative study in pear remains unknown. However, bZIP genes were divided into 13 subfamilies 

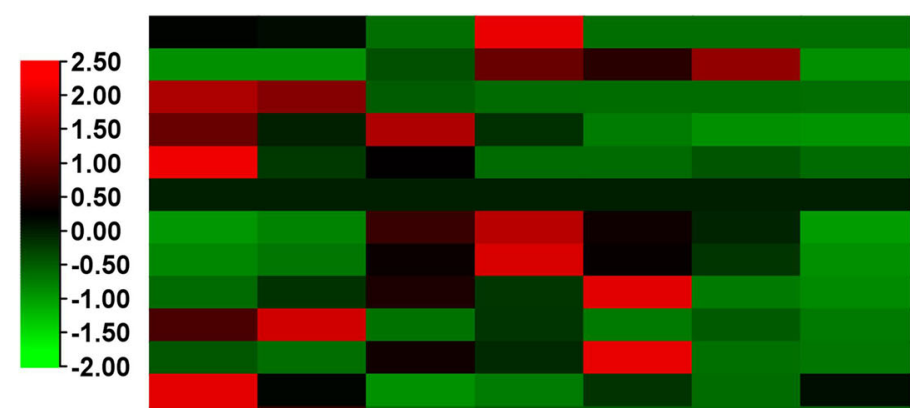

PbbZIP54 PbbZIP29 PbbZIP38 PbbZIP11 PbbZIP40 PbbZIP19 PbbZIP50 PbbZIP4 PbbZIP46 M PbbZIP2 PbbZIP53 PbbZIP33 PbbZIP9 PbbZIP39 PbbZIP51 PbbZIP6 PbbZIP45 PbbZIP23 PbbZIP35 PbbZIP30 PbbZIP13 PbbZIP1 PbbZIP56 PbbZIP42 PbbZIP18 PbbZIP43 PbbZIP27 PbbZIP17 PbbZIP52 PbbZIP55

PbbZIP31 PbbZIP14 PbbZIP57 PbbZIP3 PbbZIP25 PbbZIP37 PbbZIP58 PbbZIP36 PbbZIP41 PbbZIP12 PbbZIP10 PbbZIP48 PbbZIP44 PbbZIP32 PbbZIP15 PbbZIP20 PbbZIP21 PbbZIP60 PbbZIP8 PbbZIP34 PbbZIP49 PbbZIP59 PbbZIP24 PbbZIP26 PbbZIP47 PbbZIP61 PbbZIP5 PbbZIP62 PbbZIP16 PbbZIP28 PbbZIP22 \begin{tabular}{l|l} 
PbbZIP7 & E
\end{tabular}

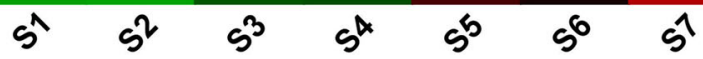




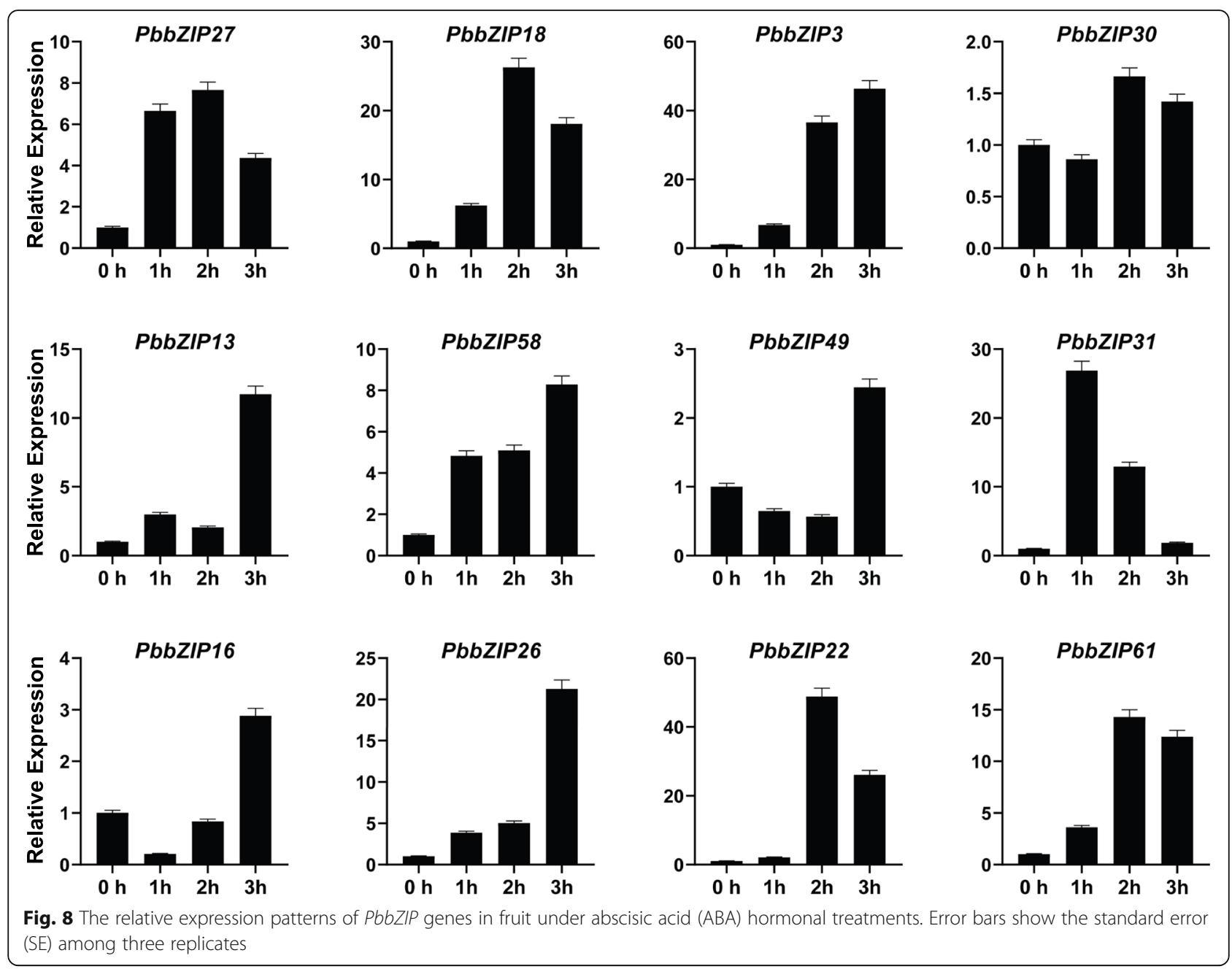

(A, B(F/Ara) $, \mathrm{C}(\mathrm{D} /$ Ara $), \mathrm{D}, \mathrm{E}(\mathrm{B} /$ Ara $), \mathrm{F}(\mathrm{H} /$ Ara $), \mathrm{G}$, $\mathrm{H}(\mathrm{E} /$ Ara $), \mathrm{I}(\mathrm{I} /$ Ara $), \mathrm{J}(\mathrm{G} /$ Ara $), \mathrm{K}(\mathrm{A} /$ Ara $), \mathrm{L}(\mathrm{C} / /$ Ara $), \mathrm{M}(\mathrm{S} /$ Ara) based on the phylogenetic tree and Arabidopsis groups. A phylogenetic tree was constructed of bZIP genes by neighbor-joining methods (NJ-M). The evolutionary analysis of the tree showed that subfamily $\mathrm{C}, \mathrm{D}$, $\mathrm{G}$, and $\mathrm{H}$ genes might be lost in the subfamily and expanded during the evolutionary mechanism in Chinese pear (Fig. 1). The number of bZIP genes in Arabidopsis, poplar, and Prunus persica was higher, while less than in gapes, apple, and strawberry as compared to a pear. These results indicate that the expansion of bZIP genes in pear. Different modes of gene duplication events (WGD-whole genome duplication, TD-tandem duplication, TRD-transposed duplication, and DSD- dispersed duplication) are the major source of driving force in the expansion and evolution process [58-60] for example, Hsf, and F-box gene family [61, 62]. Whole-genome duplication (WGD) can create a large number of duplicate genes in a short time [63]. In this present study, we identified four modes of duplication (DSDs, WGDs,
TDs, and TRDs) in the PbbZIP gene family, 42 gene pairs in whole-genome duplication, 41 pairs in dispersed duplication, 2 tandem duplication, and 2 transposed duplications. In addition, WGD and DSD genes were involved in high proportion, indicating that these modes (WGD and DSD) may play a critical role in the evolution and expansion of PbbZIPs.

Furthermore, the pairwise synonymous (Ks) and nonsynonymous $(\mathrm{Ka})$ values were calculated in paralogous gene pairs, as shown in Fig. 4 and Table S5. The pairwise $\mathrm{ka} / \mathrm{ks}$ value indicates that three types of selection (positive, purifying selection, and neutral selection). $\mathrm{Ka} / \mathrm{ks}$ value of mostly paralogous genes indicates that purifying selection in PbbZIPs gene family. Tandem duplication events contained only two gene pairs, indicating that tandem duplication was not an important role in the expansion of PbZIP genes as compared to dispersed duplication (Table S5). Additionally, we analyzed the orthologous syntenic relationship between Pyrus bretschneideri, Fragaria vesca, Prunus mume, and Prunus persica. Pyrus bretschneideri and Fragaria vesca have 63 
orthologous gene pairs, followed by Pyrus bretschneideri and Prunus mume have 61 orthologous gene pairs, while Pyrus bretschneideri and Prunus persica contained 72 orthologous gene pairs. These findings of collinearity analysis indicate that Rosaceae species (pear, peach, apple, and strawberry) strong similar syntenic orthologous and evolutionary relationship. Subsequently, the organization of gene structure (intron/exon) might imply that evolutionary trajectory in different bZIPs [64]. The amount of introns determines the plants potential to adapt and the development process [25].. Furthermore, the exon-intron compositions of bZIP individuals from the same group are frequently identified, as shown in Fig. 3. In certain gene families, this phenomenon is considered an evolutionary imprint, resulting in the development of functionally different paralogs $[65,66]$. Those genes that have shorter introns or without introns created an activation role in evolutionary selection [67]. Some PbbZIP genes, especially PbbZIP11, 40, 19, 4, and 46 , were prevalently lack of introns (Fig. 3), which could reduce the posttranscriptional processes for response to multiple abiotic stresses [68]. In this study, a maximal number of introns/exons (18/19) and minimal intron/ exon (1/1) (Fig. 3). Moreover, 20 motifs were detected in PbbZIPs through the MEME webtool. The composition of motifs was shown in Table S4. These conserved motifs composition and different numbers indicate that the functional divergence between different subfamilies. Highly conserved motif 2 was distributed in most of the genes. These results suggest that conserved motifs may play various roles in determining specific functions of bZIP proteins [25].

Varieties of cis-elements were divided into three groups; 1) related to stress (LTR, G-box, and ARE), 2) related to phytohormones (GARE motif, TGACG motif, P-box, ABRE, and CGTCA-motif), and 3) related to plant growth/development (Box 4, CAT-box, 02-site) has been carried out on the promoter regions of PbbZIPs. Transcription factors are involved in stress response, for example, ABA [69], ethylene [35], light signaling [37, 38], hormones signaling [34, 35, 36], drought [46], cold stress [41, 42] and mechanical and osmotic stress [43]. OsABF2 is positively regulating Abiotic stress and ABA signaling in rice [70]. SlbZIP38 gene identified in tomato is a response to salt stress [71]. ZjbZIPs play roles in development under abiotic and biotic stresses [5]. 10 TGA family genes in Arabidopsis play important roles in the SA defense signaling pathway and necrotizing pathogens $[5,72]$. Plants improve their biotic and abiotic response after exogenous application of gibberellin, ethylene, SA, and ABA [73]. Proteins homologous to the FD (follower locus D; ATbZIP14) and FDP (FD paralog; ATbZIP27) transcription factors in A. thaliana were found in the FD-like clades, and they may play important roles in blooming. Finally, the qRT-PCR analysis was used to determine the expression profile of PbbZIPs in fruit under exogenous hormonal stress (ABA, SA, and MeJA). PbbZIPs genes significantly showed peaked expression, except one gene (PbZIP22) on different time duration in pear fruit (Fig. 8). Based on RNA- seq data, stage1 and stage4 genes are highly expressed. bZIP genes in many plant species, including tomato [74], apple [75], and watermelon [76], have been involved in fruit development and the ripening process. However, whether bZIPs are involved in Chinese pear fruit development and post-harvest ripening is unknown. In this present study, we found that PbbZIP genes were abundantly expressed on different development stages. These results imply that PbbZIPs genes are extensively involved in fruit development and ripening processes in Chinese white pear Figs. 9 and 10.

\section{Conclusion}

In conclusion, A total of 62 PbbZIPs were identified in Chinese white pear, based on phylogenetic evolution, conserved motifs, and introns/exons analysis. PbbZIPs genes were categorized into 13 subfamilies. Wholegenome duplication (WGD) and dispersed duplication (DSD) might be highly contributed to the expansion of PbbZIPs. Moreover, chromosomal location, subcellular localization, synteny analysis, characteristics, calculate of $\mathrm{ka} / \mathrm{ks}$ value, promoter regions, gene structure analysis. Finally, qRT-PCR results showed that PbbZIPs had a significant role in abiotic and biotic stress. Our results provide us a strong base for PbbZIPs roles in fruit development, ripening process, molecular mechanism, and evolutionary relationship, especially under multiple stress conditions.

\section{Methods}

\section{Plant material and treatments}

The fruit samples 39 days after flowering were collected from a 42-year-old pear plant, which was grown in a research horticulture orchard in Dangshan, Anhui, China. The exogenous hormonal sprayed on the whole surface of fruits with $200 \mu \mathrm{M}$ salicylic acid (SA), $500 \mu \mathrm{M}$ abscisic acid (ABA), and $500 \mu \mathrm{M}$ Methyl jasmonate (MeJA) according to a previously described method [50, 77]. All fruit samples were harvested at 39 DAF and collected on $0 \mathrm{~h}, 1 \mathrm{~h}, 2 \mathrm{~h}, 3 \mathrm{~h}$. Immediately all fruit samples were frozen in liquid nitrogen and transformed into $-80^{\circ} \mathrm{C}$ for storage until further experiments.

\section{Identification, conserved domain, and sequence analysis} The whole-genome sequence of Chinese white pear along with GFF3 (general feature format file) and CDS (Coding sequences) was downloaded from the pear genome project online web server (http://peargenome.njau. 


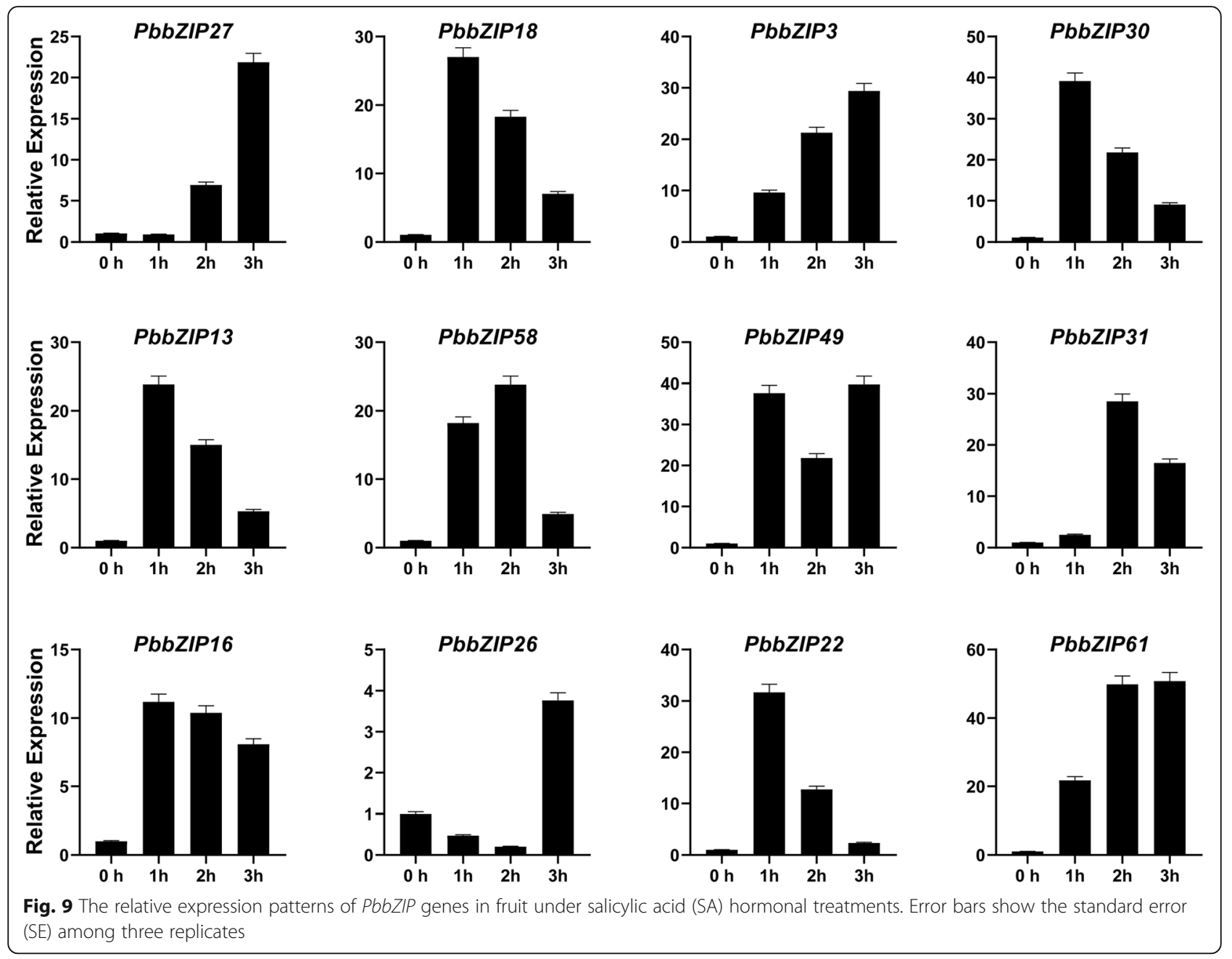

edu.cn) [78]. Furthermore, the Arabidopsis bZIP protein sequence was downloaded from the TAIR web server (http://arabidopsis.org) and Blasted against the Chinese pear genome for carryout PbbZIP genes. Secondly, the Hidden Markov Model (HMM) of the bZIP (PF00170) domain was retrieved from the Pfam database (http://pfam. xfam.org) [79], and Blast was used for the extraction of bZIP transcription factors genes from the pear genome. HMMER v3.3.2 software package and NCBI web-based conserved domain (http://ncbi.nlm.nih.gov/structure/ bwrpsb/pwrpssb.cgi) was performed with default parameters for the identification of integrity domain. Finally, all output candidate genes were verified using SMART (http://smart.embl-heidelberg.de) [80] and InterPro (htt:// www.ebi.ac.uk/InterPro/search/sequence) [81].

Gene structure and conserved motif

The MEME-suit (Multiple EM for Motif Elicitation) online tools (http://meme-suit.org) were visualized and identified the conserved motif with the following default parameters; the minimum width 6 residues and maximum width 50 residues and the maximum number of 20 motifs were identified [82]. Subsequently, the introns/exons analysis of PbZIP genes was found through general feature format (GFF3) files and visualized by using (GSDS v2.0) Gene Structure Display Server (http://gsds-gao-lab.org) [83].

\section{Chromosomal distribution and gene features}

The information about starting and ending points of each bZIP protein was investigated from the pear annotation GFF3 files the position of genes displayed using MapChart v2.3 (http://www.wur.nl/en/shoe/Mapchart. htm) [84]. Afterward, the molecular weight (MW), Isoelectric point (pI), and length of the amino acid were computed using the online web tool ExPasy (http:// www.expasy.org) [85].

Gene duplications, synteny analysis, and calculation of $\mathrm{ka} / \mathrm{ks}$ value

we used the Multiple collinearity Scan toolkit (MCScanx) [86] to examine the four modes of gene 


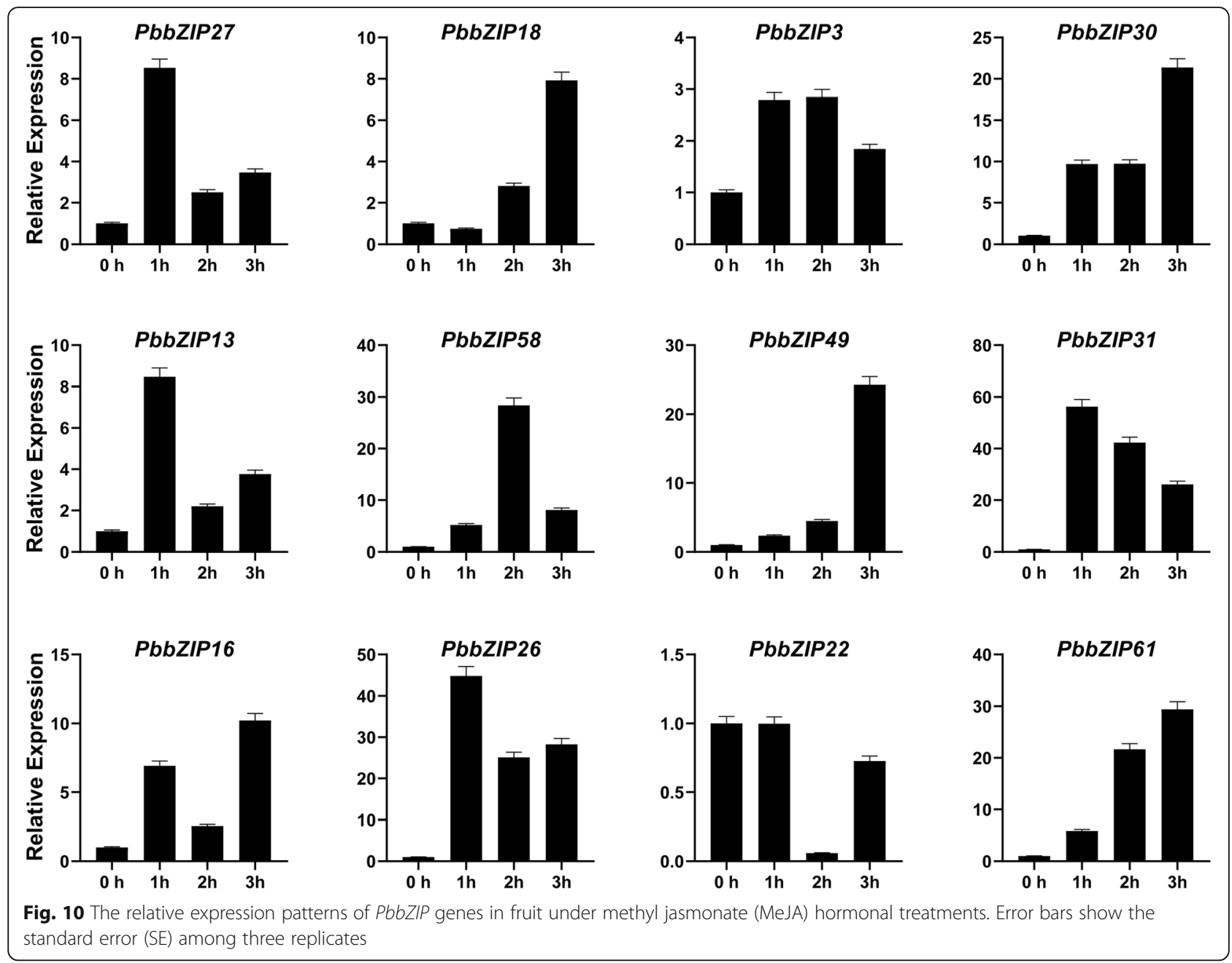

duplication (WGD, TD, TRD, and DSD) in PbbZIP genes and the synteny relationship between Pyrus bretschneideri, Fragaria vesca, Prunus mume, and Prunus persica. Finally, gene duplication and collinearity relationship displayed using circos software and Tbtool software [87] with default parameters. The ka/ks values were determined for the measurement of selection pressure during the expansion and evolution process of different gene pairs. The ka/ks values were obtained using $\mathrm{ka} / \mathrm{ks}$ calculator (http://github.com/qiaoxin/scripts_for_ Gb/tree/master /calculator_ka_ks_pipeline) [88].

\section{Cis-element analysis and gene ontology}

The potential cis-regulatory elements on promoter regions of bZIP genes were analyzed. The promoter sequence of each bZIP was used, which is already downloaded from the pear genome project (http:// peargenome.njau.edu.cn). Furthermore, the plantCare [89] web tool (http:///bioinformatics.psb.ugent.be/webtool/plantcare) to identify the putative cis-regulatory elements on promoter region. In the predicted genes, GO annotation and subcellular localization were determined by using the CELLOGO tool [90].

\section{Phylogenetic tree}

A multiple protein sequence alignment (75 Arabidopsis, 86 poplar, 55 grapevine) was aligned with the full-length amino acid of bZIP members using MEGA-X software (http://www.megasoftware.net) [91]. A phylogenetic tree was constructed with neighbor-joining methods (NJ-M) with 1000 bootstrap replicates, the pairwise deletion and Poisson correction distance method were selected with others default parameters. On the other hand, alignments were used to construct a phylogenetic tree with a maximum likelihood method using the online IQ-TREE program. Finally, the phylogenetic tree was visualized using itol software (https://itol.embl.de/) [92].

\section{Insilico expression analysis}

To investigate the expression patterns of PbbZIP genes in different fruit development stages (stage1-15DAB, stage2-30DAB, stage3-55DAB, stage4-85DAB, stage5- 
115DAB, stage6- mature stage, and stage7- fruit senescence stage) of Pyrus bretschneideri and FPKM value were carried from RNA-seq SRA (sequence read archive) data were downloaded from NCBI (http://www.ncbi.nlm.nih.gov) with following accession number, SRX1595645, SRX1595648, SRX1595646, SRX1595647, SRX1595651, SRX1595650, SRX1595652. Finally, the heat map of the PbbZIP family was visualized by using TBtool software [87].

\section{RNA isolation and quantitative real-time PCR analysis}

Total RNA was isolated from pear fruits using RNA-prep plant pure kit (Tiangen - Beijing). The qRT-PCR specific primers were designed by using the Beacon Designer 7 software and a specific primer sequence as shown in Table S1. The total qRT-PCR mixture was $20 \mu \mathrm{l}$ including $10 \mu \mathrm{l}$ SYBER premix Ex Taq II, $1 \mu \mathrm{l}$ forward, $1 \mu \mathrm{l}$ reverse primer, $6 \mu \mathrm{l}$ RNAase free water, and $2 \mu \mathrm{l} \mathrm{cDNA}$. The pear tubulin gene was used as an internal control (Table S1) $[78,80]$. The final expression patterns of PbbZIP genes were calculated using $2^{-\Delta \Delta C t}$ methods [93].

\section{Abbreviations}

bZIP: basic leucine zipper; SA: salicylic acid; ABA: abscisic acid; MeJA: methyl jasmonate; HMM: Hidden Markov Model; DAF: Days after flowering; DAB: day after blooming; GO: gene ontology; NJ: neighbor-joining; GSDS: gene structure display server; TF: transcription factors; MEME: Multiple Em for Motif Elicitation; MW: molecular weight; pl: isoelectric point; MCSscanX: multiple collinearity scan toolkit; FPKM: Fragments per kilobase million; CDS: coding sequence; RNA: ribonucleic acid; qRT-PCR: real-time quantitative reverse transcription PCR; gDNA: genomic DNA

\section{Supplementary Information}

The online version contains supplementary material available at https://doi. org/10.1186/s12870-021-03191-3.

Additional file 1: Table S1. Primer of PbbZIP's genes used for GRT-PCR. Additional file 2: Table S2. Molecular characterization of PbbZIP genes Family in Chinese pear genome.

Additional file 3: Table S3. Predicted Molecular function, cellular components biological process, and subcellular localization of PbbZIP gene family in Chinese pear.

Additional file 4: Table S4. Motif sequence in bZIP genes.

Additional file 5: Table S5. Modes of gene duplication events, and non-Synonymous (ka) and synonymous (ks) values in PbbZIP genes Family.

Additional file 6: Table S6. List of bZIP orthologous gene pairs identified in Fragaria vesca Prunus mume and Prunus persica.

Additional file 7: Table S7. investigation of cis-acting elements in PbbZIP's gene.

Additional file 8: Table S8. RNA-seq data of PbbZIP's gene on different development stages of pear fruit.

Additional file 9: Fig. S1. Chromosomal locations of PbZIP genes in $P$. bretschneideri.

Additional file 10: Fig. S2. The phylogenetic tree of bZIP genes in P. bretschneideri, F. vesca, P. persica, and $M$. domestica with maximum likelihood method. Different color indicates different subfamilies (A-I).

\section{Acknowledgements}

We appreciate pro. Yongping cai for his effort in revising and provided guidance on the whole manuscript.
Corresponding authors

Correspondence to Yongping Cai.

\section{Authors' contributions}

MAM conceived and designed the experiments; MMM, GL, MA, WH, HW, AS, MWR, and SR contributed reagents/materials/analysis tools; YC provided guidance on the whole manuscript. MAM wrote the article. All authors read and approved the final manuscript.

\section{Funding}

This work was performed at the school of Life Sciences, Anhui agricultural university, Hefei, China and was supported by National Natural Science Foundation of China (No. 31640068) and Natural Science Youth Foundation of Anhui Agricultural University (No. 2019zd01). These funding bodies had no role in the design of the study, collection, analysis, and interpretation of data or in writing the manuscript.

\section{Availability of data and materials}

The Chinese white pear bZIP protein sequences were collected from the pear genome project (http://peargenome.njau.edu.cn). The Arabidopsis bZIP protein sequences were downloaded from the genome sequences of the Arabidopsis information source (TAIR) database (http://www.arabidopsis.org). All bZIP transcription factor genes in grapevine (Vitis vinifera) and poplar sequence were downloaded from the latest database version (V1) of the $12 X$ assembly of grapevine genome (http://genomes.cribi.unipd.it/) and phytozome respectively. All RNA-Seq data were deposited in the NCBI SRA database under the following accession number, SRX1595645, SRX1595648, SRX1595646, SRX1595647, SRX1595651, SRX1595650, SRX1595652.

\section{Declarations}

Ethics approval and consent to participate Not applicable.

Consent for publication

Not applicable.

\section{Competing interests}

The authors declare that they have no competing interests.

\section{Author details}

${ }^{1}$ School of Life Sciences, Anhui Agricultural University, Hefei 230036, China. ${ }^{2}$ Department of Software Engineering, University of Gujrat, Lahore 54000, Pakistan. ${ }^{3}$ Department of Environment and Soil Sciences, University of Lleida, Avinguda Alcalde Rovira Roure 191, 25198 Lleida, Spain. ${ }^{4}$ College of Agronomy, Anhui Agricultural University, Hefei 230036, China. ${ }^{5}$ Co-Innovation Center for Sustainable Forestry in Southern China, Key Laboratory of Forest Genetics \& Biotechnology, Ministry of Education, College of Biology and the Environment, Nanjing Forestry University, Nanjing, China.

Received: 10 April 2021 Accepted: 28 August 2021

Published online: 09 September 2021

\section{References}

1. Jakoby M, Weisshaar B, Dröge-Laser W, Vicente-Carbajosa J, Tiedemann J, Kroj T, et al. bZIP transcription factors in Arabidopsis. Trends Plant Sci. 2002; 7(3):106-11. https://doi.org/10.1016/S1360-1385(01)02223-3.

2. Rian DM. PInTFDB : updated content and new features of the plant transcription factor database. 2010:38 October 2009:822-7.

3. Survey G, Jain M, Tyagi AK, Khurana JP. Genomic Survey and Gene Expression Analysis of the Basic Leucine Zipper Transcription Factor Family in Rice1 [ w ] [ OA ]. 2015;146:333-50.

4. Gai W, Ma X, Qiao Y, Shi B, Haq S, Li Q. Characterization of the bZIP Transcription Factor Family in Pepper ( Capsicum annuum L .): CabZIP25 Positively Modulates the Salt Tolerance. 2020;11 February:1-18.

5. Zhang Y, Gao W, Li H, Wang Y, Li D, Xue C, et al. Genome-wide analysis of the bZIP gene family in Chinese jujube ( Ziziphus jujuba. 2020::1-14

6. Umezawa T, Nakashima K, Miyakawa T, Kuromori T, Tanokura M, Shinozaki K, et al. Molecular basis of the core regulatory network in ABA responses: sensing, signaling and transport. Plant Cell Physiol. 2010;51(11):1821-39. https://doi.org/10.1093/pcp/pcq156. 
7. Yoshida T, Fujita Y, Sayama H, Kidokoro S, Maruyama K, Mizoi J, et al. AREB1, $A R E B 2$, and $A B F 3$ are master transcription factors that cooperatively regulate ABRE-dependent ABA signaling involved in drought stress tolerance and require $A B A$ for full activation. Plant J. 2010;61(4):672-85. https://doi.org/1 0.1111/j.1365-313X.2009.04092.x.

8. Yoshida T, Fujita Y, Maruyama K, Mogami J, Todaka D, Shinozaki K, et al. Four Arabidopsis AREB/ABF transcription factors function predominantly in gene expression downstream of SnRK2 kinases in abscisic acid signalling in response to osmotic stress. Plant Cell Environ. 2015;38(1):35-49. https://doi. org/10.1111/pce.12351.

9. Howell SH. Endoplasmic reticulum stress responses in plants. Annu Rev Plant Biol. 2013;64(1):477-99. https://doi.org/10.1146/annurev-arplant050312-120053.

10. Liu JX, Srivastava R, Howell SH. Stress-induced expression of an activated form of AtbZIP17 provides protection from salt stress in Arabidopsis. Plant Cell Environ. 2008;31(12):1735-43. https://doi.org/10.1111/j.1365-3040.2008. 01873.x.

11. Hartmann L, Pedrotti L, Weiste C, Fekete A, Schierstaedt J, Göttler J, et al. Crosstalk between two bZIP signaling pathways orchestrates salt-induced metabolic reprogramming in arabidopsis roots. Plant Cell. 2015;27(8):224460. https://doi.org/10.1105/tpc.15.00163.

12. Wang Z, Cheng K, Wan L, Yan L, Jiang H, Liu S, et al. Genome-wide analysis of the basic leucine zipper (bZIP) transcription factor gene family in six legume genomes. BMC Genomics. 2015;16(1):1-15. https://doi.org/10.1186/ s12864-015-2258-X

13. Sun $X$, Li Y, Cai H, Bai X, Ji W, Ding $X$, et al. The Arabidopsis AtbZIP1 transcription factor is a positive regulator of plant tolerance to salt, osmotic and drought stresses. J Plant Res. 2012;125(3):429-38. https://doi.org/10.1 007/s10265-011-0448-4.

14. Zhou Y, Xu D, Jia L, Huang X, Ma G, Wang S, et al. Genome-wide identification and structural analysis of bZIP transcription factor genes in brassica napus. Genes (Basel). 2017;8.

15. Li D, Fu F, Zhang H, Song F. Genome-wide systematic characterization of the bZIP transcriptional factor family in tomato (Solanum lycopersicum L.). BMC Genomics. 2015;16(1):1-18. https://doi.org/10.1186/s12864-015-1990-6.

16. Liu X, Chu Z. Genome-wide evolutionary characterization and analysis of bZIP transcription factors and their expression profiles in response to multiple abiotic stresses in Brachypodium distachyon. BMC Genomics. 2015; 16:1-15

17. Wei KA, Chen JUAN, Wang YA, Chen YA, Chen SH, Lin YINA, et al. GenomeWide Analysis of bZIP-Encoding Genes in Maize. 2012463-76.

18. Liao Y, Wei HZ, Hao WY, Jian AT, Liu HY, Chen JZS. Soybean GmbZIP44, GmbZIP62 and GmbZIP78 genes function as negative regulator of ABA signaling and confer salt and freezing tolerance in transgenic Arabidopsis. 2008::225-40

19. Xu Y, Wang Y, Zhao H, Wu M, Zhang J, Chen W, et al. Genome-wide identification and expression analysis of the bZIP transcription factors in the Mycoparasite Coniothyrium minitans. 2020.

20. Liu J, Chen N, Chen F, Cai B, Santo SD, Tornielli GB, et al. Genome-wide analysis and expression profile of the bZIP transcription factor gene family in grapevine (Vitis vinifera). 2014.

21. Zhang Z, Cui H, Xing S, Liu X, Chen X, Wang X. Computer Science \& Systems Biology Genome-Wide Identification and Analysis of bZIP Family and their Expression in Response to Multiple Abiotic Stresses in Malus domestica 2017;10:32-42.

22. Wang X, Chen X, Yang T, Cheng Q, Cheng Z. Genome-Wide Identification of bZIP Family Genes Involved in Drought and Heat Stresses in Strawberry ( Fragaria vesca). 2017;2017

23. Wang J, Zhou J, Zhang B, Vanitha J, Ramachandran S, Jiang S. Genomewide Expansion and Expression Divergence of the Basic Leucine Zipper Transcription Factors in Higher Plants with an Emphasis on Sorghum 2011; 53:212-231.

24. Wang $X L$, Zhong $Y$, Cheng ZM, Xiong JS. Divergence of the bZIP gene family in strawberry, peach, and apple suggests multiple modes of gene evolution after duplication. Int J Genomics. 2015;2015:1-11. https://doi.org/1 $0.1155 / 2015 / 536943$

25. Jin Z, Xu W, Liu A. Genomic surveys and expression analysis of bZIP gene family in castor bean ( Ricinus communis L .). 2014;:299-312.

26. Guan $Y$, Ren $H$, Xie H, Ma Z, Chen F. Identification and characterization of bZIP-type transcription factors involved in carrot ( Daucus carota L .) somatic embryogenesis. 2009;:207-17.
27. Izawa T, Foster R, Nakajima M, Shimamoto K, Chua N, The S, et al. The Rice bZIP Transcriptional Activator RITA-1 Is Highly Expressed during Seed Development Published by: American Society of Plant Biologists ( ASPB ) Linked references are available on JSTOR for this article : 2015;6:1277-87.

28. Fukazawa J, Sakai T, Ishida S, Yamaguchi I, Kamiya Y. REPRESSION OF SHOOT GROWTH , a bZIP Transcriptional Activator, Regulates Cell Elongation by Controlling the Level of Gibberellins. 2000;12 June:901-15.

29. Yin Y, Zhu Q, Dai S, Lamb C, Beachy RN. RF2a, a bZIP transcriptional activator of the phloem-specific rice tungro bacilliform virus promoter, functions in vascular development. 1997;16:5247-59.

30. Ren $A D$, Cha D. AtbZIP34 is required for Arabidopsis pollen wall patterning and the control of several metabolic pathways in developing pollen. 2009;: 581-601.

31. Liu J, Srivastava R, Che P, Howell SH, Co-laboratory RJC, la A. Salt stress responses in Arabidopsis utilize a signal transduction pathway related to endoplasmic reticulum stress signaling 2007;3:897-909.

32. Light PU, Huang X, Ouyang X, Yang P, Lau OS, Li G, et al. FHY3 and HY5 Positively Mediate Induction Arabidopsis of COP1 Transcription in Response to Photomorphogenic UV-B LightP ${ }^{\text {тм. }}$. 2015;24:4590-606.

33. Finkelstein RR, Lynch TJ. Abscisic acid inhibition of radicle emergence but not seedling growth is suppressed by sugars. Plant Physiol. 2000;122(4): 1179-86. https://doi.org/10.1104/pp.122.4.1179.

34. Nieva C, Busk PK, Domı E, Lumbreras V. Isolation and functional characterisation of two new bZIP maize regulators of the ABA responsive gene rab28. 2005,:899-914.

35. Li Z, Zhang L, Yu Y, Quan R, Zhang Z, Zhang H, et al. The ethylene response factor AtERF11 that is transcriptionally modulated by the bZIP transcription factor HY5 is a crucial repressor for ethylene biosynthesis in Arabidopsis. 2011;5:88-99.

36. Zander M, Chen S, Imkampe J, Thurow C, Gatz C. Repression of the Arabidopsis thaliana Jasmonic acid / ethylene-induced defense pathway by TGA-interacting Glutaredoxins depends on their C-terminal ALWL motif. 2012; December 2011:1-10.

37. Ulm R, Baumann A, Oravecz A. Genome-wide analysis of gene expression reveals function of the bZIP transcription factor HY5 in the UV-B response of Arabidopsis. 2004;101:1397-402.

38. Osterlund MT, Hardtke CS, Wei N, Deng XW. Targeted destabilization of HY5 during light-regulated development of Arabidopsis. 2000;405:0-4.

39. Pontier D, Miao Z, Lam E. Trans-dominant suppression of plant TGA factors reveals their negative and positive roles in plant defense responses 2001;27.

40. Thurow C, Schiermeyer A, Krawczyk S, Butterbrodt T, Nickolov K, Gatz C, et al. Tobacco bZIP transcription factor TGA2 . 2 and related factor TGA2 . 1 have distinct roles in plant defense responses and plant development. 2005 1:100-13.

41. Shimizu H, Sato K, Berberich T, Miyazaki A, Ozaki R, Imai R, et al. LIP19, a Basic Region Leucine Zipper Protein, is a Fos-like Molecular Switch in the Cold Signaling of Rice Plants. 2005:46:1623-34.

42. Liu C, Wu Y, Wang X. bZIP transcription factor OsbZIP52 / RISBZ5 : a potential negative regulator of cold and drought stress response in rice. 2012::1157-69.

43. Wang X, Chaban AC, Hanson ÆJ, Teige ÆM, Harter ÆKK, Sjef JVA, et al. Expression patterns within the Arabidopsis C / S1 bZIP transcription factor network : availability of heterodimerization partners controls gene expression during stress response and development. 2009;1:107-19.

44. Liu G, Wang J, Cramer G, Dai Z, Duan W, Xu H, et al. Transcriptomic analysis of grape ( Vitis vinifera $L$.) leaves during and after recovery from heat stress. 2012.

45. Yang Y, Lv W, Li M, Wang B, Sun D, Deng X, et al. Maize Membrane-Bound Transcription Factor Zmbzip17 is a Key Regulator in the Cross-Talk of ER Quality Control and ABA Signaling. 2020;1:2020-33.

46. Liu C, Mao B, Ou S, Cl-channel R. OsbZIP71 , a bZIP transcription factor , confers salinity and drought tolerance in rice. 2013.

47. Orellana S, Yañez M, Espinoza A, Verdugo I, González E, Ruiz-Lara S, et al. The transcription factor SIAREB1 confers drought, salt stress tolerance and regulates biotic and abiotic stress-related genes in tomato. Plant Cell Environ. 2010; 33(12):2191-208. https://doi.org/10.1111/j.1365-3040.2010.02220.x

48. Bastías A, Yañez M, Osorio S, Arbona V, Gómez-Cadenas A, Fernie AR, et al. The transcription factor AREB1 regulates primary metabolic pathways in tomato fruits. J Exp Bot. 2014;65(9):2351-63. https:/doi.org/10.1093/jxb/eru114.

49. Su X, Zhao Y, Wang H, Li G, Cheng X, Jin Q, et al. Transcriptomic analysis of early fruit development in Chinese white pear (Pyrus bretschneideri Rehd.) and functional identification of PbCCR1 in lignin biosynthesis. BMC Plant Biol. 2019;19(1):417. https://doi.org/10.1186/s12870-019-2046-X. 
50. Aamir M, Cheng X, Li G, Su X, Abdullah M, Cai Y. Gene structure, evolution and expression analysis of the P-ATPase gene family in Chinese pear ( Pyrus bretschneideri ). Comput Biol Chem. 2020;88:107346. doi:https://doi.org/10.1 016/j.compbiolchem.2020.107346.

51. Qiao X, Yin H, Li L, Wang R, Wu J, Wu J, Zhang S. Different modes of gene duplication show divergent evolutionary patterns and contribute differently to the expansion of gene families involved in important fruit traits in pear (Pyrus bretschneideri). Front Plant Sci. 2018;9:161. https://doi.org/10.3389/ fpls.2018.00161.

52. Abdullah M, Cheng X, Cao Y, Su X, Manzoor MA, Gao J, Cai Y, Li, Y. Zinc fingerhomeodomain transcriptional factors (ZHDs) in upland cotton (Gossypium hirsutum): Genome-wide identification and expression analysis in fiber development. Front Genet. 2018;9:357. https://doi.org/10.3389/fgene.2018.00357.

53. Cheng X, Li M, Abdullah M, Li G, Zhang J, Manzoor MA, Wang H, Jin Q, Jiang T, Cai Y, Li D. In silico genome-wide analysis of the pear (Pyrus bretschneideri) KNOX family and the functional characterization of PbKNOX1, an arabidopsis Brevipedicellus orthologue gene, involved in cell wall and lignin biosynthesis. Front Genet. 2019;10:632. https://doi.org/10.3389/fgene.2019.00632.

54. Abdullah $M$, Cao $Y$, Cheng $X$, Meng $D$, Chen $Y$, Shakoor $A$, et al. The sucrose synthase gene family in chinese pear (pyrus bretschneideri Rehd.): structure, expression, and evolution. Molecules. 2018;23:1-16.

55. Abdullah M, Cao Y, Cheng X, Shakoor A, Su X. Genome-Wide Analysis Characterization and Evolution of SBP Genes in Fragaria vesca, Pyrus bretschneideri, Prunus persica and Prunus mume. 2018:1-12.

56. Balbontín C, Gutiérrez C, Wolff M, Figueroa CR. Effect of abscisic acid and methyl jasmonate preharvest applications on fruit quality and cracking tolerance of sweet cherry. 2018;78 September:438-46.

57. Cheng X, Muhammad A, Li G, Zhang J, Cheng J, Qiu J, Jiang T, Jin Q, Cai Y, Lin Y Family-1 UDP glycosyltransferases in pear ( Pyrus bretschneideri ): Molecular identification, phylogenomic characterization and expression profiling during stone cell formation. Mol Biol Rep 2019;0:0. doi:https://doi. org/10.1007/s11033-019-04669-y, 46, 2, 2153, 2175.

58. Doerks T, Copley RR, Ponting CP, Bork P. Systematic Identification of Novel Protein Domain Families Associated with Nuclear Functions. 2002:47-56.

59. Moore RC, Purugganan MD. The early stages of duplicate gene evolution; 2003.

60. Cao Y, Han Y, Meng D, Abdullah M, Yu J, Li D, et al. Expansion and evolutionary patterns of GDSL-type esterases/lipases in Rosaceae genomes. Funct Integr Genomics. 2018;18(6):673-84. https://doi.org/10.1007/s10142-018-0620-1.

61. Zhang S, Tian Z, Li H, Guo Y, Zhang Y, Roberts JA, et al. Genome-wide analysis and characterization of F-box gene family in Gossypium hirsutum L. 2019::1-16.

62. Qiao X, Li M, Li L, Yin H, Wu J, Zhang S. Genome-wide identification and comparative analysis of the heat shock transcription factor family in Chinese white pear (Pyrus bretschneideri) and five other Rosaceae species. BMC Plant Biol. 2015;15:1-16.

63. Wang $Y$, Wang $X$, Paterson AH. Genome and gene duplications and gene expression divergence : a view from plants. 2012;:1-14.

64. Azeem F, Tahir H, ljaz U, Shaheen T. RESEARCH ARTICLE A genome-wide comparative analysis of bZIP transcription factors in $\mathrm{G}$. arboreum and $\mathrm{G}$ raimondii ( Diploid ancestors of present-day cotton ). Physiol Mol Biol Plants. 2020. doi:https://doi.org/10.1007/s12298-020-00771-9.

65. Li X, Gao S, Tang Y, Li L, Zhang F, Feng B, et al. Genome-wide identification and evolutionary analyses of bZIP transcription factors in wheat and its relatives and expression profiles of anther development related TabZIP genes. BMC Genomics. 2015;16(1):1-21. https://doi.org/10.1186/s12864-015-2196-7.

66. Liu H, Zhong Y, Guo C, Wang XL, Xiong J, Cheng Q, et al. Genome-wide analysis and evolution of the bZIP transcription factor gene family in six Fragaria species. Plant Syst Evol. 2017;303(9):1225-37. https:/doi.org/10.1007/s00606-017-1433-8.

67. Zhu M, Yan B, Hu Y, Cui Z, Wang X. Genomics genome-wide identification and phylogenetic analysis of rice FTIP gene family. Genomics. 2020;112(5): 3803-14. https://doi.org/10.1016/j.ygeno.2020.03.003.

68. Zhou Y, Zeng L, Chen R, Wang Y, Song J. Genome-wide identification and characterization of stress-associated protein (SAP) gene family encoding A20/AN1 zinc-finger proteins in Medicago truncatula. Arch Biol Sci. 2018; 70(1):87-98. https://doi.org/10.2298/ABS170529028Z.

69. Choi H, Hong J, Ha J, Kang J, Kim SY. ABFs, a Family of ABA-responsive Element Binding Factors * a large number of ABA / stress-responsive genes in. 2000;275:1723-30.

70. Hossain A, Cho J, Han M, Ahn C, Jeon J, An G, et al. The ABRE-binding bZIP transcription factor OsABF2 is a positive regulator of abiotic stress and ABA signaling in rice. J Plant Physiol. 2010;167(17):1512-20. https://doi.org/10.101 6/j.jplph.2010.05.008.
71. Pan Y, Hu X, Li C, Xu X, Su C, Li J, Song H, Zhang X, Pan, Y. SlbZIP38, a tomato bZIP family gene downregulated by abscisic acid, is a negative regulator of drought and salt stress tolerance. Genes. 2017;8(12):402. https:// doi.org/10.3390/genes8120402.

72. Peng Y, Sun T, Zhang Y. Perception of Salicylic Acid in Physcomitrella patens. Front Plant Sci. 2017:8:2145. https://doi.org/10.3389/fpls.2017.02145.

73. Wani SH, Kumar V, Shriram V. Sah SK. NU SC Crop J. 2016:4(3):162-76. https://doi.org/10.1016/j.cj.2016.01.010.

74. Rohrmann J, Tohge T, Alba R, Osorio S, Caldana C, McQuinn R, et al. Combined transcription factor profiling, microarray analysis and metabolite profiling reveals the transcriptional control of metabolic shifts occurring during tomato fruit development. Plant J. 2011;68(6):999-1013. https://doi. org/10.1111/j.1365-313X.2011.04750.x.

75. Costa F, Alba R, Schouten H, Soglio V, Gianfranceschi L, Serra S, et al. This Provisional PDF corresponds to the article as it appeared upon acceptance. Fully formatted Use of homologous and heterologous gene expression profiling tools to characterize transcription dynamics during apple fruit maturation and ripening Article t. 2010;:1-17.

76. Levi A, Davis A, Hernandez A, Wechter P, Thimmapuram J, Trebitsh T, et al. Genes expressed during the development and ripening of watermelon fruit. Plant Cell Rep. 2006;25(11):1233-45. https://doi.org/10.1007/s00299-006-0163-0.

77. Li G, Liu X, Zhang Y, Muhammad A, Han W, Li D, et al. Plant Physiology and Biochemistry Cloning and functional characterization of two cinnamate 4hydroxylase genes from Pyrus bretschneideri. Plant Physiol Biochem. 2020; 156:135-45. doi:https://doi.org/10.1016/j.plaphy.2020.07.035.

78. Wu J, Wang Z, Shi Z, Zhang S, Ming R, Zhu S, et al. The genome of the pear (Pyrus bretschneideri Rehd.). Genome Res. 2013;23(2):396-408. https://doi. org/10.1101/gr.144311.112.

79. Finn RD. Pfam: clans, web tools and services. Nucleic Acids Res. 2006; 34(90001):D247-51. https://doi.org/10.1093/nar/gkj149.

80. Letunic I, Doerks T, Bork P. SMART 7: recent updates to the protein domain annotation resource. Nucleic Acids Res. 2012;40:302-5.

81. Zdobnov EM, Apweiler R. InterProScan - An integration platform for the signature-recognition methods in InterPro. Bioinformatics. 2001;17(9):847-8. https://doi.org/10.1093/bioinformatics/17.9.847.

82. Bailey TL, Boden M, Buske FA, Frith M, Grant CE, Clementi L, et al. MEME S UITE : tools for motif discovery and searching; 2009. p. 1-7.

83. Hu B, Jin J, Guo A, Zhang H, Luo J. Genome analysis GSDS 2 . 0 : an upgraded gene feature visualization server. 2015;31 December 2014:1296-7.

84. Voorrips RE. Computer Note MapChart : Software for the Graphical Presentation of Linkage Maps and QTLs. 1994;:77-8.

85. Artimo P, Jonnalagedda M, Arnold K, Baratin D, Flegel V, Fortier A, et al. EXPASy: SIB bioinformatics resource portal. 2012;40:597-603.

86. Wang Y, Tang H, Debarry JD, Tan X, Li J, Wang X, et al. MCScanX: a toolkit for detection and evolutionary analysis of gene synteny and collinearity. Nucleic Acids Res. 2012:40:1-14.

87. Chen C, Chen H, Zhang Y, Thomas HR, Frank MH. TBtools - an integrative toolkit developed for interactive analyses of big biological data. Mol Plant. 2020;13(8):1194-202. https://doi.org/10.1016/j.molp.2020.06.009.

88. Wang D, Zhang Y, Zhang Z, Zhu J, Yu J. KaKs Calculator 2.0: a toolkit incorporating gamma-series methods and sliding window strategies. Genomics. Proteomics Bioinforma. 2010;8:77-80.

89. Lescot M. PlantCARE, a database of plant cis-acting regulatory elements and a portal to tools for in silico analysis of promoter sequences. Nucleic Acids Res. 2002:30(1):325-7. https://doi.org/10.1093/nar/30.1.325.

90. Yu CS, Chen YC, Lu CH, Hwang JK. Prediction of protein subcellular localization. Proteins: Structure, Function, and Bioinformatics. 2006;64(3):64351. https://doi.org/10.1002/prot.21018..

91. Kumar S, Stecher G, Li M, Knyaz C, Tamura K. Brief Communication ( Revised) MEGA X : molecular evolutionary genetics analysis across computing platforms. Mol Biol Evol. 2018;35(6):1547. https://doi.org/10.1093/molbev/msy096.

92. Letunic I, Bork P. Interactive tree of life (iTOL) v4: recent updates and new developments. Nucleic Acids Res. 2019;47(W1):W256-9. https://doi.org/10.1 093/nar/gkz239.

93. Livak KJ, Schmittgen TD. Analysis of relative gene expression data using real-time quantitative $P C R$ and the 2- $\Delta \Delta C T$ method. Methods. 2001;25(4): 402-8. https://doi.org/10.1006/meth.2001.1262.

\section{Publisher's Note}

Springer Nature remains neutral with regard to jurisdictional claims in published maps and institutional affiliations. 